\title{
Identification of Potential Indicators for Survival in Patients With Thyroid Cancer Based on Mrna Expression Levels of Family With Sequence Similarity 3 Members
}

\author{
Yuting Ma \\ Affiliated Hospital of Weifang Medical University \\ Junfeng Shi \\ Affiliated Hospital of Weifang Medical University \\ Yongping Liu \\ Affiliated Hospital of Weifang Medical University \\ Ruiyan Pan \\ Weifang Medical University \\ Hongyan Qiu \\ Weifang Medical University \\ Fang Han \\ Weifang Medical University \\ Ningning Hou \\ Weifang Medical University
}

Xiaodong Sun ( $\square$ xiaodong.sun@wfmc.edu.cn )

Department of Endocrinology, Affiliated Hospital of Weifang Medical University, 2428 Yuhe Road, Weifang, Shandong, 261031, China https://orcid.org/0000-0001-7775-2823

\section{Research}

Keywords: thyroid cancer, biomarker, bioinformatics analysis, tumor microenvironment, immunity

Posted Date: September 21st, 2020

DOl: https://doi.org/10.21203/rs.3.rs-75597/v1

License: (c) (1) This work is licensed under a Creative Commons Attribution 4.0 International License. Read Full License 


\section{Abstract}

Background: Thyroid cancer (THCA) is a common head and neck malignancy. The family with sequence similarity 3 (FAM3) is a cytokine-like gene family with four members, which is presumed to participate in the development of many cancer types. However, the expression patterns of FAM3s in THCA and their prognostic values, have not yet been established.

Methods: We investigated differential expressions of FAM3 mRNA and protein in THCA, then validated the findings for FAM3B by immunohistochemistry. We also investigated survival data with respect to FAM3 expression patterns in patients with THCA. FAM3s information regarding their relationships with clinical pathological parameters were obtained and FAM3 mutations were assessed. KEGG and GO pathway regarding FAM $3 \mathrm{C}$ were obtained using online databases. To investigate potential correlations between FAM3s and immune cell infiltration, we investigated the roles of FAM3s in immune cells of patients with THCA.

Results: The mRNA expression of FAM3C were significantly elevated in THCA tissues; high expression levels of FAM3C protein were also observed in THCA tissues. A significant association between the pathological stage and the expression of FAM3C was found in patients with THCA. Patients with THCA who had high mRNA expression levels of FAM3C exhibited significantly more favourable prognosis, compared with patients who had low mRNA expression levels of FAM3C.

Conclusions: Overall, FAM3C may play vital roles in the pathogenesis and development of THCA, and these findings constitute novel insights for biomarkers of immunotherapeutic targeted agents and may aid in the identification of prognostic biomarkers for THCA.

\section{Introduction}

Thyroid carcinoma (THCA) constitutes more than $90 \%$ of all endocrine malignancies [1]. Recently, THCA was identified as the cancer with the most rapidly increasing global incidence in the past two decades [1]. Notably, papillary thyroid carcinoma (PTC) is the malignancy with the most rapidly increasing incidence worldwide. It has been reported that approximately $15 \%$ of PTC patients relapse within 10 years after initial treatment, leading to invasive disease and poor survival outcomes [2]. From 2000 to 2016, the incidence of aggressive PTC variants significantly increased, compared with well-differentiated PTC [3]. There are obvious differences in the comparative prognosis and survival results for various mutations in PTC, compared with well-differentiated and anaplastic subtypes [3]. Consequently, it is important to investigate new biomarkers associated with survival and prognosis in patients with THCA.

The family with sequence similarity 3 (FAM3) is a cytokine-like gene family with four members, which was initially characterised in 2002. These four genes (FAM3A, FAM3B, FAM3C, and FAM3D) were considered to be a new family of genes because the homology among them ranged from 31.6-53.3\%, while they did not exhibit significant homology with other known proteins [4]. Over the past few decades, 
there has been increasing evidence that members of the FAM3 have essential roles in the occurrence and development of a number of diseases, such as cancer and diabetes.

Previous research has shown that FAM3 members are expressed in a variety of cancers, such as colon cancer [5] and oesophageal carcinoma [6]. However, the identification of FAM3s as treatment targets and prognostic biomarkers for THCA has not been reported. With the rapid development of sequencing technology (e.g., next generation sequencing technology), various databases have been established, which enable a comprehensive analysis of FAM3s.

In this study, we explored new biomarkers for use as therapeutic targets and in prognostic prediction of THCA. In particular, we performed a deep and complete bioinformatics analysis of the expression levels of FAM3s in THCA to evaluate their potential as prognostic biomarkers based on copy numbers published online, thus providing additional data for selection of adequate treatment and prediction of long-term outcomes for patients with THCA.

\section{Materials And Methods}

\section{UALCAN}

UALCAN is an interactive web resource that can be used to analyse the relative mRNA expression levels of genes between tumours and normal samples [7]. UALCAN can also be used to explore associations of mRNA expression levels with relative clinicopathologic parameters. In the present study, "TCGA Analysis" data were obtained from UALCAN; then, expression levels of FAM3s and their relationships with clinical pathological parameters were determined. $p<0.05$ was considered statistically significant.

\section{Human Protein Atlas}

The Human Protein Map contains immunohistochemistry data regarding 20 extremely common types of cancer [8]. To identify specific proteins differentially expressed in THCA, comparisons of protein expression levels of each FAM3 between normal human tissues and tissues from patients with THCA were performed using immunohistochemistry images obtained from the "Tissue" and "PATHOLOGY" modules of the Human Protein Atlas, respectively.

\section{Kaplan-meier Plotter}

The Kaplan-Meier plotter software can provide graphical representations of the associations between gene expression and survival in patients with various cancers, including breast cancer and liver cancer. [9, 10]. The prognostic values of mRNA expression levels of each FAM3 member in THCA were analysed by using the Kaplan-Meier plotter software. In this analysis, patients with THCA were separated into high and low expression groups, depending on the median mRNA expression levels, then assessed by Kaplan- 
Meier survival plots; median mRNA expression levels, number-at-risk cases, hazard ratios (HR) with 95\% confidence intervals (Cls), and log rank p values. $p<0.05$ was considered statistically significant.

\section{Cbioportal}

cBioPortal is a comprehensive web resource that can explore, visualise, and analyse multidimensional cancer genomics data $[11,12]$. This study analysed the genomic profiles of FAM3s in THCA, which contained the genetic mutations, copy-number changes from the GISTIC (Genomic Identification of Significant Targets in Cance), and mRNA expression z-Scores (RNASeq V2 RSEM, z-score threshold \pm 2.0 ). Mutations of FAM3s and their relationships with OS and DFS of patients with THCA were investigated using Kaplan-Meier plots. The log-rank test was used to compare survival curves. $p<0.05$ was considered statistically significant.

\section{Genecards}

GeneCards is an online open access website resource of the human gene compendium, which enables comparisons of relationships among human genes, variants, proteins, and biological pathways [13]. Functions of FAM3C significantly associated with THCA were analysed by GO and KEGG in the GeneCards database. GO enrichment analysis (including biological processes [BPs], cellular components [CCs], and molecular functions [MFs]) was used to predict the functional roles of FAM3C, and KEGG analysis was used to define pathways associated with FAM3C.

\section{Uniprot}

The UniProt knowledgebase is a long-term collection of databases, which contains arrays of sequences and functional information regarding various proteins [14]. BPs, CCs, and MF were identified by GO enrichment, while KEGG pathways were obtained by UniProt analysis.

\section{TIMER}

TIMER is a reliable, intuitional tool for systematic evaluation regarding infiltration of various immune cells and their clinical effects [15]. This study evaluated relationships of FAM3s with immune cell infiltration and clinical outcomes, using the "Gene module" and "Survival module," respectively.

\section{Immunohistochemistry}

Cryosections of THCA tissues and normal thyroid tissues were obtained and stained with haematoxylin and eosin. An antibody against FAM3B (abs119356, Absin Bioscience Inc.) was used for immunohistochemical staining, in accordance with the manufacturer's instructions. 


\section{Results}

\section{mRNA and protein levels of FAM3s in patients with THCA}

This study was performed to elucidate the latent molecular mechanisms of FAM3 in patients with THCA. Analyses of mRNA and protein levels were performed by using the UALCAN database and the Human Protein Atlas, respectively. mRNA expression levels of four FAM3 members in PTC and normal thyroid tissues were measured by the UALCAN database, as shown in Fig. 1. mRNA expression levels of FAM3A were downregulated in PTC tissues, compared with normal tissues $(p=0.043$, Fig. $1 \mathrm{~A})$. Furthermore, mRNA expression levels of FAM3B were significantly downregulated in gland papillary carcinoma tissues $(p<0.001$, Fig. 1B). Significant upregulation of FAM3C and D mRNA expression levels was observed in thyroid gland papillary carcinoma tissues, compared with normal samples $(p<0.001$, Fig. 1C, D).

Protein levels of FAM3s in THCA tissues were analysed using the Human Protein Atlas. Figure 2 shows that FAM $3 A$ and FAM3B exhibited intermediate protein expression levels in both normal thyroid tissues and THCA tissues (Fig. 2A-D). FAM3C exhibited intermediate protein expression levels in normal thyroid tissues, but high expression levels in THCA tissues (Fig. 2E, F). Furthermore, FAM3D exhibited low protein expression levels in normal thyroid tissues, but no expression in THCA tissues (Fig. 2G, H).

Overall, our results showed that FAM3C was overexpressed at both mRNA and protein levels in THCA tissues. Considering the significant difference in mRNA expression level of FAM3B between THCA tissues and normal thyroid tissues, we validated the FAM3B protein expression data reported in the Human Protein Atlas. For this validation, we repeated the immunohistochemical analysis of FAM3B, which revealed that the FAM3B protein level was enhanced in THCA tissues (Fig. 3). On the basis of the two results of immunohistochemistry of FAM3B, no definite conclusions could be drawn regarding the use of FAM3B as a biomarker in the pathogenesis of THCA.

\section{Relationships of FAM3 mRNA expression levels with clinicopathological parameters in patients with THCA}

Next, the relationships of FAM3 mRNA levels with clinicopathological parameters of patients with THCA were analysed using UALCAN, including an analysis of individual patients' cancer stages. Figure 4 shows that the mRNA expression levels of FAM3B, C, and D were significantly associated with various stages of cancer (Fig. 4B-D). In particular, patients with more advanced stages of cancer tended to have lower mRNA levels of FAM3B; the lowest mRNA level of FAM3B was observed in patients with stage 2 THCA. Furthermore, the mRNA expression level of FAM3B significantly down-regulated in stages 2 compared with stages 1 of THCA; similar results were observed regarding stages 2 and 3 (Fig. 4B). Patients with more advanced stages of cancer tended to have higher mRNA levels of FAM3C; the highest mRNA level of FAM3C was observed in patients with stage 3 THCA (Fig. 4C). In summary, these results suggested that mRNA expression levels of FAM3B, C, and D were closely associated with clinicopathological parameters in patients with THCA. 
Kaplan-Meier plotter software was used to analyse prognostic values of FAM3 mRNA expression levels in patients with THCA. As shown in Fig. 5, mRNA expression levels of FAM3B, C, and D were significantly associated with prognosis in patients with THCA. First, the association between the combined mRNA level of all four FAM3s and the prognosis of patients with THCA was analysed (Fig. 5A). Notably, the combined mRNA level of all four FAM3s was not associated with overall survival (OS) in patients with THCA (HR $=2,95 \% \mathrm{Cl}: 0.720-5.51$, and $p=0.170)$. Subsequently, associations between the mRNA levels of distinct FAM3s and the prognosis of patients with THCA were analysed. As shown in Fig. 5E and G, higher mRNA expression levels of FAM3B (HR $=0.110,95 \% \mathrm{Cl}: 0.020-0.480$, and $p<0.001)$ and FAM3C $(\mathrm{HR}=0.260,95 \% \mathrm{Cl}: 0.1-0.7$, and $p=0.004)$ were significantly associated with favourable OS in patients with THCA, while a higher mRNA expression level of FAM3D was associated with shorter OS in patients with THCA (HR $=2.92,95 \% \mathrm{Cl}: 1.08-7.90$, and $p=0.027)$ (Fig. 5I). Furthermore, the combined mRNA expression level of FAM3A, B, C, and D was associated with disease-free survival (DFS) in patients with THCA (HR $=0.380,95 \% \mathrm{Cl}: 0.170-0.860$, and $p=0.016$ ) (Fig. 5B). Additionally, associations between mRNA expression levels of distinct FAM3s and DFS in patients with THCA were analysed. Figure 5 shows that a higher mRNA expression level of FAM3B was associated with shorter DFS in patients with THCA $(\mathrm{HR}=0.460,95 \% \mathrm{Cl}: 0.210-0.990$, and $p=0.043, \mathrm{HR}=2.49,95 \% \mathrm{Cl}: 1.15-5.39$, and $p=0.016)$ (Fig. 5D, F). These results revealed that mRNA expression levels of FAM3C were significantly associated with prognosis in patients with THCA and might be useful as biomarkers to predict survival of these patients.

\section{Mutations in FAM3s and their relationships with OS and DFS in patients with THCA}

Genetic alterations in FAM3s and their relationships with OS and DFS in patients with THCA were performed by Kaplan-Meier plots. Figure 6A shows that FAM3s had low mutation rates in patients with THCA. Among 497 sequenced patients with THCA, only 78 exhibited genetic alterations; thus, the mutation rate was $16 \%$. The mutation rates of $F A M 3 A, B, C$, and $D$ were $6 \%, 4 \%, 5 \%$, and $1.8 \%$, respectively; the FAM3D gene exhibited the lowest mutation rate. Furthermore, results of Kaplan-Meier and log-rank analyses showed that genetic mutations in FAM3s were not significantly associated with OS and DFS in patients with THCA (Fig. 6B, C).

\section{Functional Enrichment Analysis Of Fam3c In Patients With Thca}

The functions of differentially expressed FAM3C were analysed by means of GeneCards and UniProt. Table 1 shows the most highly enriched GO items and KEGG pathways. GO enrichment analysis predicts the functions of target genes from three main perspectives: CCs, BPs, and MFs. Among the highly enriched functions in the BP class, platelet degranulation, signal transduction, multicellular organism development, and negative regulation of gluconeogenesis were associated with the occurrence and development of THCA. In the CC class, the extracellular region, Golgi apparatus, platelet dense granule 
lumen, cytoplasmic vesicle, and extracellular exosome were enriched, in association with the occurrence and development of THCA. In the MF class, cytokine activity and protein binding were enriched in association with the occurrence and development of THCA. Additionally, four pathways associated with the functions of FAM3C in THCA were observed by KEGG analysis. Among these KEGG pathways, the response to elevated platelet cytosolic $\mathrm{Ca}^{2+}$ was significantly associated with THCA tumorigenesis and progression (Table 1).

Table 1

The enrichment analysis of different expressed FAM3C in THCA (GeneCards and Unprot), including $\mathrm{GO}$ enrichment in cellular component terms, biological process terms, and molecular function terms and KEGG enriched terms.

\begin{tabular}{|lll|}
\hline Term ID & Function & Details \\
\hline R-HSA-109582.2 & pathway & Platelet activation, signaling and aggregation \\
R-HSA-76005.2 & pathway & Response to elevated platelet cytosolic Ca2+ \\
R-HSA-114608.3 & pathway & Platelet degranulation \\
\hline R-HSA-109582.2 & pathway & Hemostasis \\
\hline GO:0002576 & BP & platelet degranulation \\
\hline GO:0007165 & BP & signal transduction \\
\hline GO:0007275 & BP & multicellular organism development \\
\hline GO:0008150 & BP & biological_process \\
\hline GO:0045721 & BP & negative regulation of gluconeogenesis \\
\hline GO:0005576 & CC & extracellular region \\
\hline GO:0005794 & CC & Golgi apparatus \\
\hline GO:0031089 & CC & platelet dense granule lumen \\
\hline GO:0031410 & CC & cytoplasmic vesicle \\
\hline GO:0070062 & CC & extracellular exosome \\
\hline GO:0005125 & MF & cytokine activity \\
\hline GO:0005515 & MF & protein binding \\
\hline
\end{tabular}

\section{Infiltration of FAM3s in immune cells of patients with THCA}

FAM3s have been shown to participate in the infiltration of immune cells and inflammatory responses, thereby affecting the prognosis of patients with THCA. Thus, we comprehensively explored correlations between differentially expressed FAM3s and immune cell infiltration using the TIMER database. There were negative correlations between the mRNA expression level of FAM3A and infiltration by $\mathrm{B}$ cells (Cor = 
- 0.309, $p<0.001), \mathrm{CD} 8+\mathrm{T}$ cells (Cor $=-0.145, p<0.001), \mathrm{CD} 4+\mathrm{T}$ cells $(\mathrm{Cor}=-0.313, p<0.001)$, macrophages (Cor $=-0.358, p<0.001$ ), neutrophils (Cor $=-0.513, p<0.001)$, and dendritic cells (Cor $=-$ $0.514, p<0.001$; Fig. 7A). However, the mRNA expression level of FAM3B was positively correlated with the infiltration of $\mathrm{B}$ cells (Cor $=0.178, p<0.001)$, CD4 + T cells (Cor $=0.205, p<0.001)$, macrophages (Cor $=0.230, p<0.001$ ), neutrophils (Cor $=0.268, p<0.001)$, and dendritic cells (Cor $=0.299, p<0.001 ;$ Fig. 7B). A negative correlation was observed between the mRNA expression level of FAM3C and the infiltration of CD8 $+\mathrm{T}$ cells (Cor $=-0.378, p<0.001$ ), whereas positive correlations were observed with the infiltration of B cells (Cor $=0.409, p<0.001), \mathrm{CD} 4+\mathrm{T}$ cells (Cor $=0.392, p<0.001)$, macrophages (Cor $=0.356, p<$ 0.001), neutrophils (Cor $=0.281, p<0.001$ ), and dendritic cells (Cor $=0.214, p<0.001$; Fig. 7 C). Moreover, the mRNA expression level of FAM3D was positively correlated with the infiltration of CD8 + T cells (Cor = $0.101, p=0.026$ ), whereas it was negatively correlated with the infiltration of B cells (Cor $=-0.228, p<$ $0.001)$, CD $4+\mathrm{T}$ cells (Cor $=-0.257, p<0.001)$, macrophages (Cor $=-0.213, p<0.001)$, neutrophils (Cor $=$ $-0.242, p<0.001$ ), and dendritic cells (Cor $=-0.214, p<0.001$; Fig. 7D). Correlations were also assessed between differentially expressed FAM3s and infiltration of immune cells. Cox proportional hazard models were used to adjust for the following confounding factors: B cells, CD $8+T$ cells, CD $4+T$ cells, macrophages, neutrophils, dendritic, FAM3A, FAM3B, FAM3C, FAM3D, and cancer stage. CD $8+$ T cells ( $p$ $=0.017)$, dendritic cells $(p=0.047)$, age $(p=0.000)$, and stage 4 cancer $(p=0.039)$ were associated with prognosis in patients with THCA (Table 2). 
Table 2

The cox proportional hazard model of FAM3s and six tumor-infiltrating immune cells in THCA (TIMER).

\begin{tabular}{|llllllll|}
\hline & coef & HR & 95\%CI_I & $95 \%$ Cl_u & p.value & sig \\
\hline Age & 0.214 & 1.238 & 1.122 & 1.37 & 0 & $* * *$ \\
\hline Stage2 & 0.050 & 1.051 & 0.075 & 14.6 & 0.971 & \\
\hline Stage3 & 1.506 & 4.51 & 0.387 & 52.6 & 0.229 & \\
\hline Stage4 & 3.183 & 24.127 & 1.17 & 498 & 0.039 & $*$ \\
\hline Gender male & -0.254 & 0.776 & 0.146 & 4.12 & 0.766 & \\
\hline Race Black & 15.649 & $6.25 * 10^{6}$ & 0 & Inf & 0.998 & \\
\hline Race White & 15.081 & $3.54 * 10^{6}$ & 0 & Inf & 0.998 & \\
\hline Purity & 6.836 & 931.2 & 11.541 & $7.51 * 10^{4}$ & 0.002 & $* *$ \\
\hline B_cell & 1.901 & 6.695 & 0 & $2.42 * 10^{6}$ & 0.771 & \\
\hline CD8_Tcell & -24.859 & 0 & 0 & 0.012 & 0.017 & $*$ \\
\hline CD4_Tcell & 7.247 & 1403.8 & 0.009 & $2.18 * 10^{8}$ & 0.235 & \\
\hline Macrophage & -29.770 & 0 & 0 & 5.25 & 0.063 & \\
\hline Neutrophil & -47.797 & 0 & 0 & $7.03 * 10^{9}$ & 0.184 & \\
\hline Dendritic & 16.608 & $1.63 * 10^{7}$ & 1.22 & $2.18 * 10^{14}$ & 0.047 & $*$ \\
\hline FAM3A & 1.968 & 7.159 & 0.607 & 84.5 & 0.118 & \\
\hline FAM3B & -0.202 & 0.817 & 0.429 & 1.56 & 0.539 & \\
\hline FAM3C & 0.711 & 2.035 & 0.522 & 7.93 & 0.306 & \\
\hline FAM3D & -0.612 & 0.542 & 0.112 & 2.62 & 0.447 & \\
\hline$* P<0.05$, & & & & & & \\
\hline$* * P<0.01$, & & & & & & \\
\hline$* * * P 0.001$ & & & & & & & \\
\hline
\end{tabular}

\section{Discussion}

FAM3s were initially reported to have important roles in the progression of insulin resistance, diabetes, and non-alcoholic fatty liver disease [16-18]; they were also identified as important regulators of liver glucose and lipid metabolism. However, the biological function and prognostic value of FAM3s in THCA 
have remained unclear. This study analysed the mRNA/protein expression levels, mutation statuses, and prognostic values of different FAM3s in THCA.

FAM3A is a novel mitochondrial protein. To the best of our knowledge, there have been no reports of FAM3A and cancer. Our results showed that mRNA expression levels of FAM3A were downregulated in THCA. FAM3B is known to be specifically synthesised and secreted by pancreatic islets; thus, FAM3B is considered a pancreatic derivative factor. In the past 10 years, FAM3B has been reported to play essential roles in the progression of various cancers, including prostate, gastric, oral, and colon carcinomas $[5,6]$. Surprisingly, some studies have shown that FAM3B is overexpressed in colon and prostate carcinomas, thereby promoting the invasion and migration of colon cancer cells, as well as the occurrence and progression of prostate cancer $[5,19]$. A relatively recent study revealed that FAM3B was expressed at a significantly lower level in oral squamous cell carcinoma [20]. Our study also showed that the mRNA expression level of FAM3B was downregulated in THCA. These observations indicate that expression levels of FAM3B vary among types of cancer. FAM3C expression is reportedly elevated in gastric cancer tissue, thereby promoting epithelial-mesenchymal transition (EMT), as demonstrated by enhanced expression of Snail and Slug, along with reduced expression of E-cadherin [21]. FAM3C is regarded as a carcinogenic factor, which plays important roles in breast cancer metastasis and EMT [22]. Consistent with the results of prior studies, we found that FAM $3 C$ was highly expressed in thyroid tumours. In the present study, lower mRNA expression levels of FAM3D were found in THCA tissues. Importantly, a prior study reported that FAM3D could be detected in peripheral blood samples; thus, it might be a suitable diagnostic marker for early colon cancer [23].

However, higher mRNA levels of FAM3B and FAM3C were significantly associated with favourable OS in patients with THCA. Initially, FAM3B was identified as a trigger for cell apoptosis and was therefore considered a potential tumour biomarker [24]. Thus far, FAM3B has been reportedly associated with tumour progression in several studies. However, the FAM3C protein, also known as leukocyte interleukinlike EMT-inducing agent, is considered a necessary and sufficient factor to cause EMT, slow down tumour growth, and prevent the onset of late stages of metastasis [25]. Our results also showed that a more advanced tumour stage was associated with a lower mRNA expression level of FAM3B and a higher mRNA expression level of FAM3C. Thus, FAM3B may serve as an oncogene in THCA, while FAM3C constitutes a tumour suppressor gene.

In this study, we also investigated protein levels of FAM3s. The results showed that FAM $3 \mathrm{C}$ proteins were highly expressed in THCA tissues, which indicated that FAM3C proteins have been shown to play important roles in embryo development, EMT, and the onset of pancreatic cancer [26, 27]. We identified a cluster of > 30 genes specific for "complete EMT" and metastasis, including FAM3C; all participated in human cancer development. However, a definite tumourigenic effect of FAM3C in THCA was not observed in the past study. Therefore, the exact role of FAM3C in THCA requires further investigation.

Notably, our study showed conflicting findings regarding the roles of FAM3B in THCA. A low FAM3B mRNA expression level was observed in THCA tissues, while a high FAM3B protein expression level was 
also observed in those tissues. Furthermore, FAM3B protein exhibited intermediate expression levels in both normal thyroid tissues and THCA tissues, according to the Human Protein Atlas. This discrepancy may be related to the small sample size or an unidentified confounding factor. Therefore, a large, prospective study is needed to elucidate the role of FAM3B in THCA.

Genetic mutation is a known aetiology of cancer pathogenesis, which may affect tumour differentiation and the prognosis of disease. However, patients with THCA exhibit low rates of genetic alteration; moreover, genetic alterations in FAM3s were not significantly associated with OS or DFS in patients with THCA. These findings indicated that FAM3s are relatively stable in THCA.

Finally, we analysed FAM3C function with respect to $G 0$ enrichment and KEGG pathway enrichment. Our results indicated that FAM3C was involved in CCs, MFs, and BPs that affected platelets. Among the KEGG pathways, the response to elevated platelet cytosolic $\mathrm{Ca}^{2+}$ was identified as the main pathway involving FAM3C. Cancer is known to regulate the production of circulating platelets. In some patients, these interactions between platelets and cancer cells can inhibit the immune identification and elimination of cancer cells, supporting the survival and spread of cancer cells; this interaction constitutes an important target for prevention and treatment [28]. Moreover, signal transduction and development processes in multicellular organisms are important contributors to the growth and development of cancer. Accordingly, FAM3C may be involved in many biological processes that affect the onset and progression of THCA.

Interactions between the tumour and the host immune system are essential for the detection of prognostic biomarkers, the reduction of drug resistance, and the development of new treatment. There is increasing evidence that the progress and recurrence of THCA are affected by the infiltration of immune cells, and that this infiltration is a vital determinant of the response to immunotherapy and subsequent clinical outcomes [29]. In this study, we found that the expression level of FAM3C was significantly correlated with infiltration of immune cells, indicating that FAM $3 \mathrm{C}$ may be a useful prognostic indicator that reflects immune status.

\section{Limitations}

This study had some limitations. First, the results were obtained via re-analysis of data from the TCGA database; no mechanistic exploration was performed. Second, the protein levels of FAM3B determined in our validation experiment conflicted with the results from the Human Protein Atlas; this discrepancy requires validation by means of a broader and more systematic study. Third, the potential diagnostic and therapeutic effects of FAM3s were not evaluated in patients with THCA. Therefore, further investigations are needed to determine whether FAM3s can be used as a diagnostic marker or therapeutic target. Finally, this study did not explore potential mechanisms of each FAM3 in the onset or progression of THCA. Future studies are needed to investigate the underlying mechanisms by which each FAM3 affects THCA.

\section{Conclusion}


This study explored the four members of FAMs by means of bioinformatics analysis. The results revealed that FAM3C might be a suitable candidate for use as a diagnostic biomarker of THCA and an indicator of survival in patients with THCA. Furthermore, FAM3C was found to be associated with the stage of cancer in patients with THCA.

\section{Declarations}

\section{Ethics approval and consent to participate}

The experimental protocol was established, according to the ethical guidelines of the Helsinki Declaration and was approved by the Human Ethics Committee of Affiliated Hospital of Weifang Medical University. Written informed consent was obtained from individual or guardian participants.

\section{Consent for publication}

Not applicable.

\section{Availability of data and materials}

Datasets used and analyzed during the current study are available from the corresponding author on reasonable request.

\section{Competing interests}

The authors declare no potential conflicts of interest.

\section{Funding:}

This study was supported in part by grants from National Natural Science Foundation of China (81870593), Natural Science Foundation of Shandong Province of China (ZR2018MH008) \Shandong Province Higher Educational Science and Technology Program for Youth Innovation (2020KJL004)

\section{Authors' Contributions}

Hou and Sun: Conceptualization, Writing - Review \& Editing.

Ma and Shi: Methodology, Software, Visualization, Writing - Original Draft.

Other authors: Software.

\section{Acknowledgments}

This study was funded by the National Natural Science Foundation of China (81870593), Natural Science Foundation of Shandong Province of China (ZR2018MH008) 『Shandong Province Higher Educational Science and Technology Program for Youth Innovation (2020KJL004). 


\section{References}

1. Siegel RL, Miller KD, Jemal A. Cancer Statistics. 2017. CA Cancer J Clin. 2017;67(1):7-30.

2. de Melo TG, Zantut-Wittmann DE, Ficher E da. ALV. Factors related to mortality in patients with papillary and follicular thyroid cancer in long-term follow-up. J Endocrinol Invest. 2014;37(12):1195200.

3. Ho AS, Luu M, Barrios L, Chen I, Melany M, Ali N, et al. Incidence and Mortality Risk Spectrum Across Aggressive Variants of Papillary Thyroid Carcinoma. JAMA Oncol. 2020.

4. Zhu Y, Xu G, Patel A, McLaughlin MM, Silverman C, Knecht K, et al. Cloning, expression, and initial characterization of a novel cytokine-like gene family. Genomics. 2002;80(2):144-50.

5. Li Z, Mou H, Wang T, Xue J, Deng B, Qian L, et al. A non-secretory form of FAM3B promotes invasion and metastasis of human colon cancer cells by upregulating Slug expression. Cancer Lett. 2013;328(2):278-84.

6. He SL, Wang WP, Yang YS, Li EM, Xu LY, Chen LQ. FAM3B promotes progression of oesophageal carcinoma via regulating the AKT-MDM2-p53 signalling axis and the epithelial-mesenchymal transition. J Cell Mol Med. 2019;23(2):1375-85.

7. Chandrashekar DS, Bashel B, SAH B, Creighton CJ, Ponce-Rodriguez I, BVSK C, et al. UALCAN: A Portal for Facilitating Tumor Subgroup Gene Expression and Survival Analyses. Neoplasia. 2017;19(8):649-58.

8. Asplund A, Edqvist PH, Schwenk JM, Pontén F. Antibodies for profiling the human proteome-The Human Protein Atlas as a resource for cancer research. Proteomics. 2012;12(13):2067-77.

9. Nagy Á, Lánczky A, Menyhárt O, Győrffy B. Validation of miRNA prognostic power in hepatocellular carcinoma using expression data of independent datasets. Sci Rep. 2018;8(1):9227.

10. Győrffy B, Surowiak P, Budczies J, Lánczky A. Online survival analysis software to assess the prognostic value of biomarkers using transcriptomic data in non-small-cell lung cancer. PLoS One. 2013;8(12):e82241.

11. Gao J, Aksoy BA, Dogrusoz U, Dresdner G, Gross B, Sumer SO, et al. Integrative analysis of complex cancer genomics and clinical profiles using the cBioPortal. Sci Signal. 2013;6(269):pl1.

12. Cerami E, Gao J, Dogrusoz U, Gross BE, Sumer So, Aksoy BA, et al. The cBio cancer genomics portal: an open platform for exploring multidimensional cancer genomics data. Cancer Discov. 2012;2(5):401-4.

13. Stelzer G, Rosen N, Plaschkes I, Zimmerman S, Twik M, Fishilevich S, et al. The GeneCards Suite: From Gene Data Mining to Disease Genome Sequence Analyses. Curr Protoc Bioinformatics. 2016;54:1.30.1-1.30.33.

14. UniProt. the universal protein knowledgebase. Nucleic Acids Res. 2017;45(D1):D158-8D169.

15. Li T, Fan J, Wang B, Traugh N, Chen Q, Liu JS, et al. TIMER: A Web Server for Comprehensive Analysis of Tumor-Infiltrating Immune Cells. Cancer Res. 2017;77(21):e108-8e110. 
16. Wang C, Chi Y, Li J, Miao Y, Li S, Su W, et al. FAM3A activates PI3K p110a/Akt signaling to ameliorate hepatic gluconeogenesis and lipogenesis. Hepatology. 2014;59(5):1779-90.

17. Wang C, Burkhardt BR, Guan Y, Yang J. Role of pancreatic-derived factor in type 2 diabetes: evidence from pancreatic $\beta$ cells and liver. Nutr Rev. 2012;70(2):100-6.

18. Chen Z, Ding L, Yang W, Wang J, Chen L, Chang Y, et al. Hepatic Activation of the FAM3C-HSF1-CaM Pathway Attenuates Hyperglycemia of Obese Diabetic Mice. Diabetes. 2017;66(5):1185-97.

19. Maciel-Silva P, Caldeira I, de Assis Santos I, ACO C, Siqueira FR, Antonioli E, et al. FAM3B/PANDER inhibits cell death and increases prostate tumor growth by modulating the expression of $\mathrm{Bcl}-2$ and Bcl-XL cell survival genes. BMC Cancer. 2018;18(1):90.

20. Shiiba M, Ishige S, Saito Y, et al. Down-regulated expression of family with sequence similarity 3 , member B (FAM3B), in oral squamous cell carcinoma. Oral Sci Int. 2012;9:9-16.

21. Shi M, Duan G, Nie S, Shen S, Zou X. Elevated FAM3C promotes cell epithelial- mesenchymal transition and cell migration in gastric cancer. Onco Targets Ther. 2018;11:8491-505.

22. Woosley AN, Dalton AC, Hussey GS, Howley BV, Mohanty BK, Grelet S, et al. TGF $\beta$ promotes breast cancer stem cell self-renewal through an ILEI/LIFR signaling axis. Oncogene. 2019;38(20):3794-811.

23. Solmi R, De Sanctis P, Zucchini C, Ugolini G, Rosati G, Del GM, et al. Search for epithelial-specific mRNAs in peripheral blood of patients with colon cancer by RT-PCR. Int J Oncol. 2004;25(4):104956.

24. Yang J, Gao Z, Robert CE, Burkhardt BR, Gaweska H, Wagner A, et al. Structure-function studies of PANDER, an islet specific cytokine inducing cell death of insulin-secreting beta cells. Biochemistry. 2005;44(34):11342-52.

25. Waerner T, Alacakaptan M, Tamir I, Oberauer R, Gal A, Brabletz T, et al. ILEl: a cytokine essential for EMT, tumor formation, and late events in metastasis in epithelial cells. Cancer Cell. 2006;10(3):22739.

26. Grønborg M, Kristiansen TZ, Iwahori A, Chang R, Reddy R, Sato N, et al. Biomarker discovery from pancreatic cancer secretome using a differential proteomic approach. Mol Cell Proteomics. 2006;5(1):157-71.

27. Pilipenko VV, Reece A, Choo DI, Greinwald JH. Genomic organization and expression analysis of the murine Fam3c gene. Gene. 2004;335:159-68.

28. Menter DG, Tucker SC, Kopetz S, Sood AK, Crissman JD, Honn KV. Platelets and cancer: a casual or causal relationship: revisited. Cancer Metastasis Rev. 2014;33(1):231-69.

29. Bindea G, Mlecnik B, Tosolini M, Kirilovsky A, Waldner M, Obenauf AC, et al. Spatiotemporal dynamics of intratumoral immune cells reveal the immune landscape in human cancer. Immunity. 2013;39(4):782-95.

\section{Figures}


A

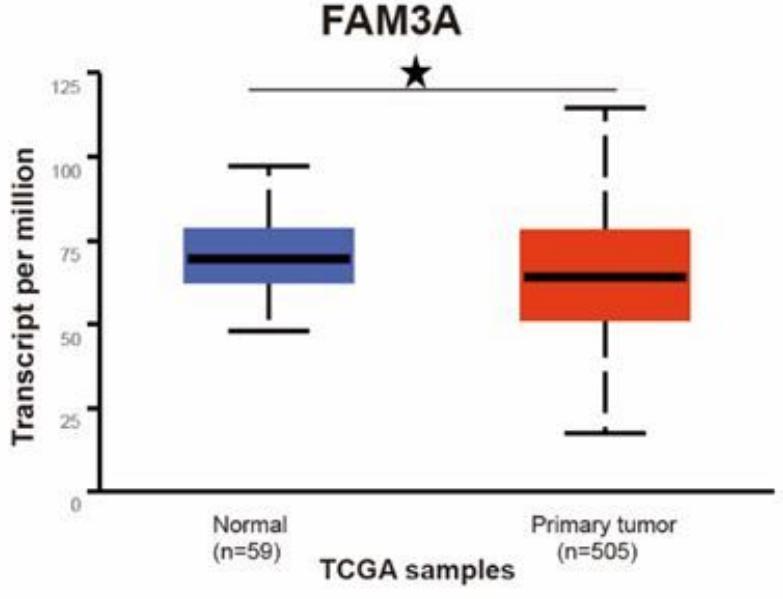

C

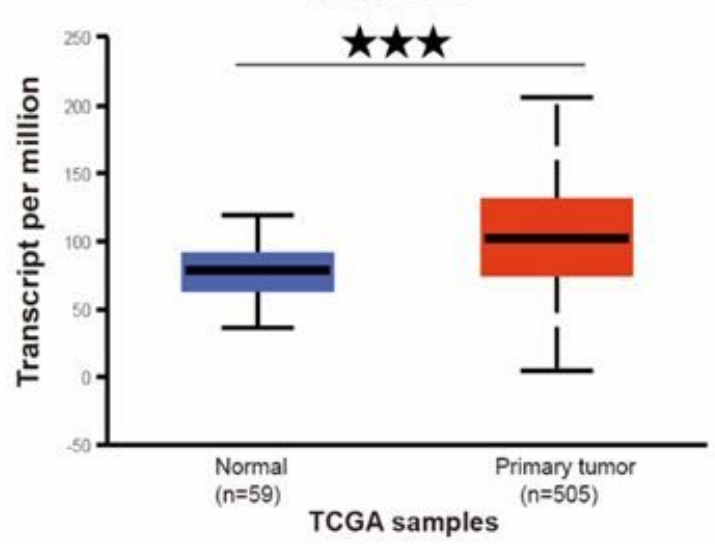

B

B FAM3B

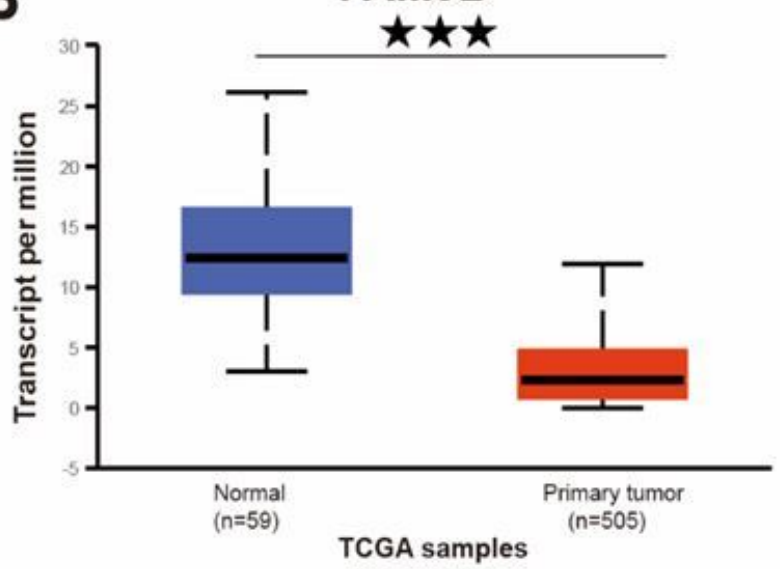

D

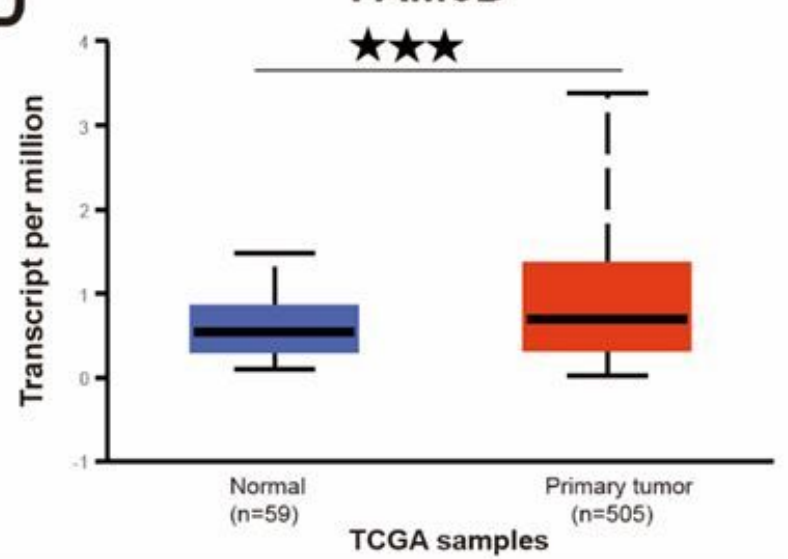

Figure 1

mRNA expression of distinct FAM3 family members in THCA tissues and adjacent normal thyroid tissues (UALCAN). mRNA expressions of FAM3A/B were found to be down-expressed in primary THCA tissues compared to normal samples (A, B). However, mRNA expressions of FAMC was found to be overexpressed in primary THCA tissues compared to normal samples (C). ${ }^{*} p<0.05,{ }^{\star *} p<0.01,{ }^{\star \star *} p<0.001$. 


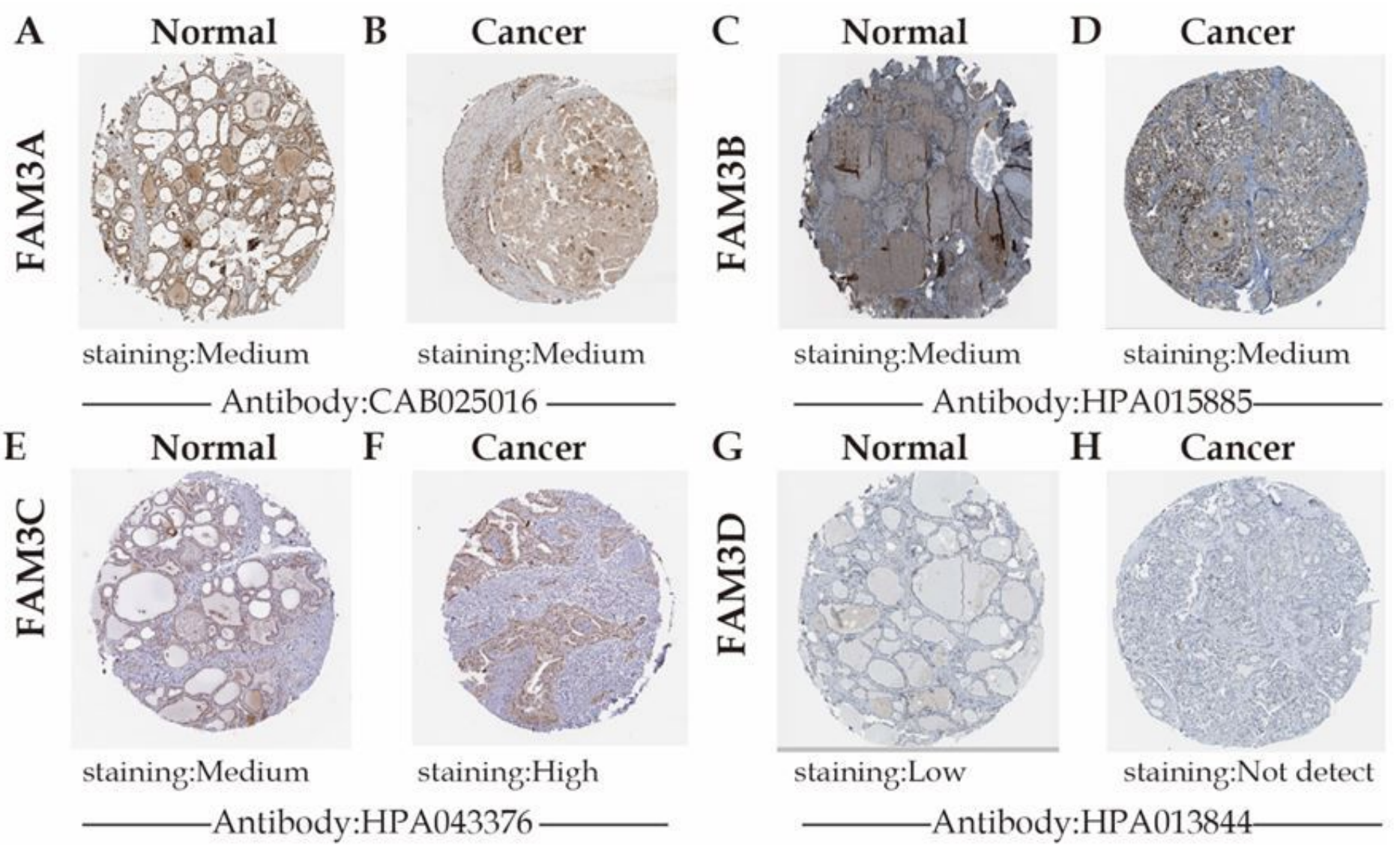

Figure 2

Representative immunohistochemistry images of distinct FAM3 family members in THCA tissues and normal thyroid tissues (Human Protein Atlas). Medium protein expression of FAM3A/B was observed both at normal thyroid tissues and THCA tissues (A-D). FAM3C proteins with medium expression in normal thyroid tissues, whereas high expressions were observed in THCA tissues (E, F). Low protein expressions of FAM3D were found in normal liver tissues, while their expressions were not detected in THCA tissues $(G, H)$. 


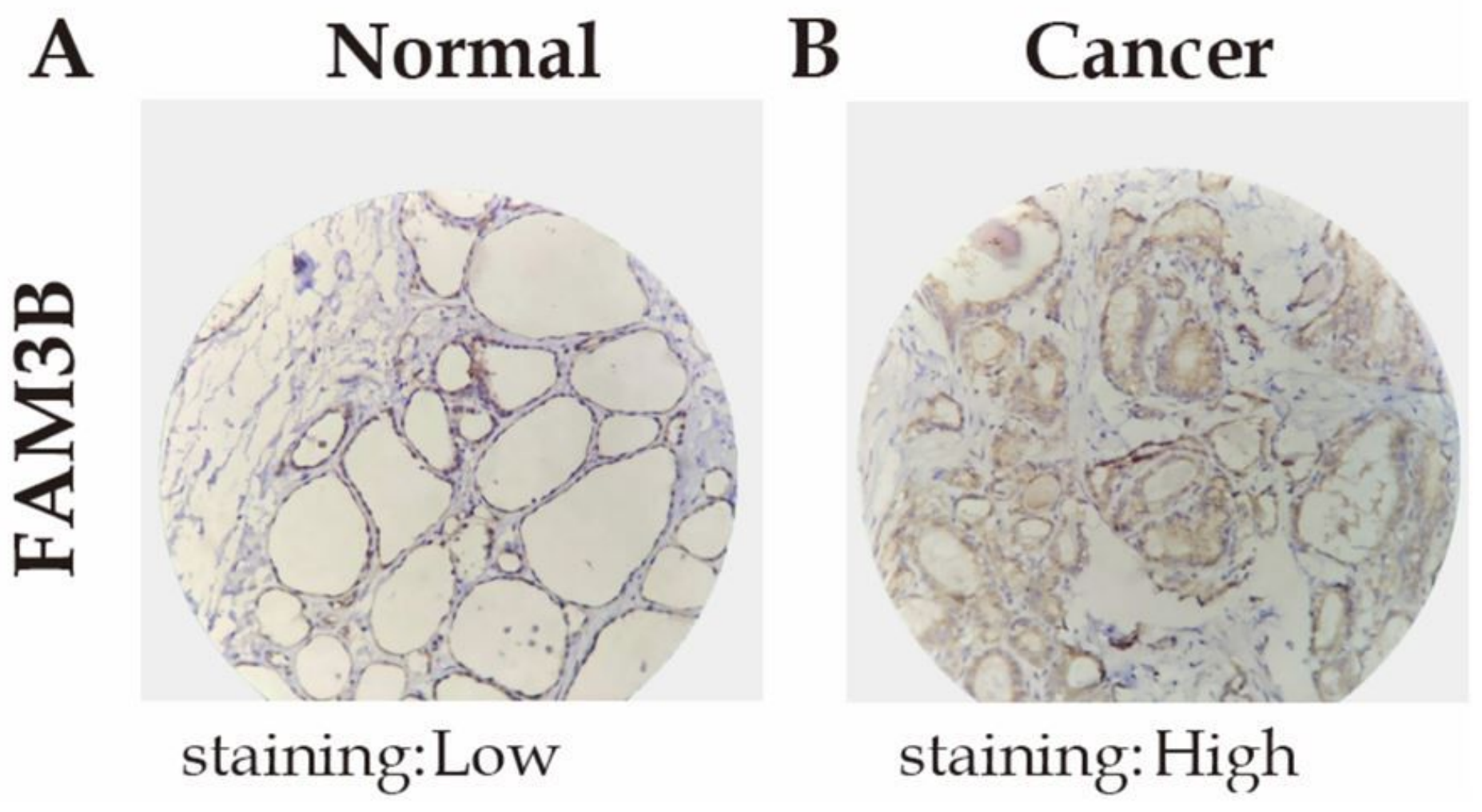

Figure 3

Expression levels for FAM3B by immunohistochemistry. FAM3B staining images of the normal thyroid tissues (A) and THCA tissues (B). 

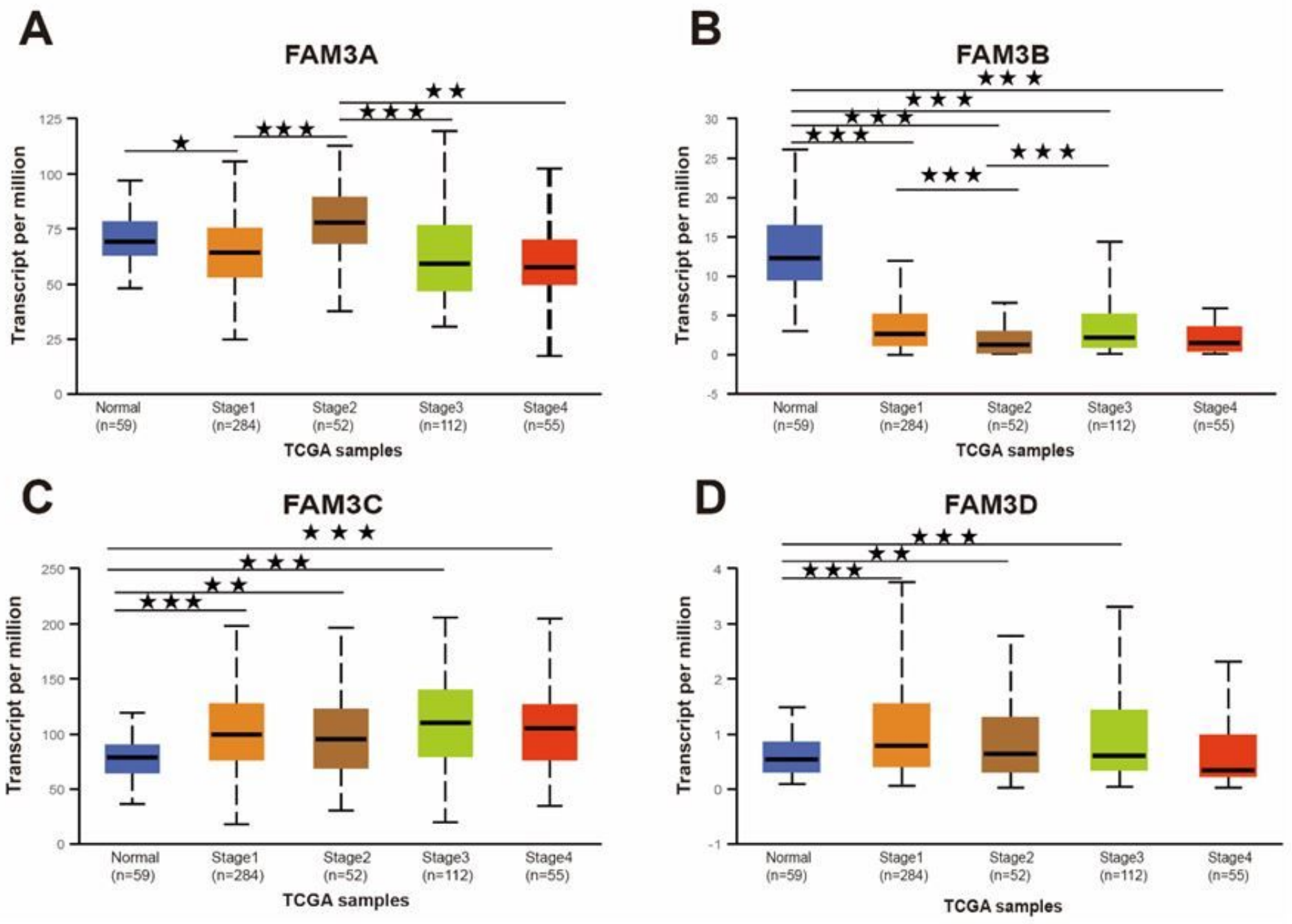

Figure 4

Relationship between mRNA expression of distinct FAM3 members and individual cancer stages of THCA patients. Patients who were in more advanced stages tended to express higher mRNA expression of FAM3C, while patients who were in more advanced stages tended to express lower mRNA expression of FAM3B. ${ }^{*} p<0.05,{ }^{*} p<0.01,{ }^{* *} p<0.001$. 

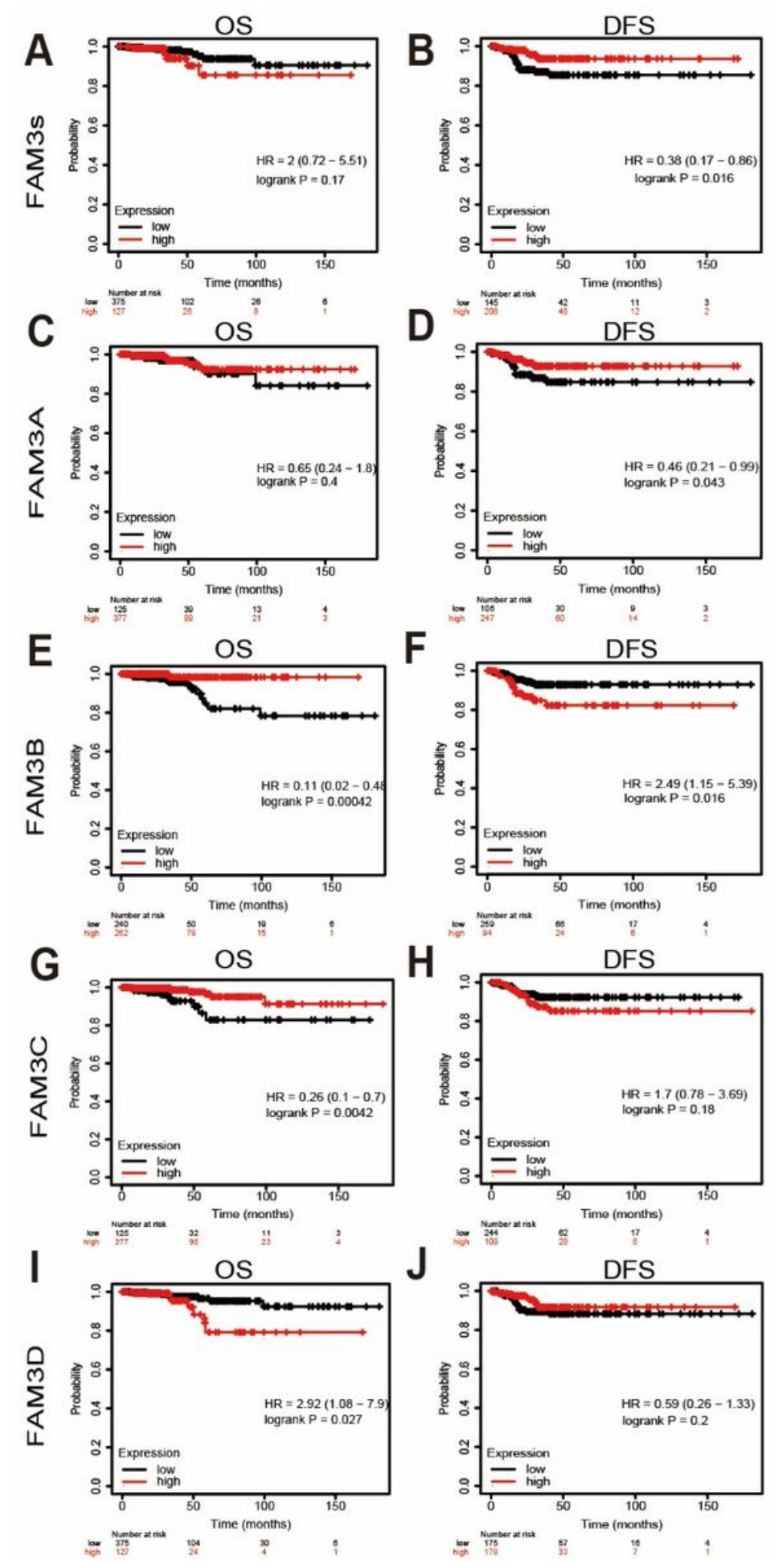

\section{Figure 5}

Prognostic value of mRNA expression of distinct FAM3 family members in thyroid cancer patients (Kaplan-MeierPlotter). Specifically, higher mRNA expressions of FAM3B/C were significantly associated with favorable $\mathrm{OS}$ of thyroid cancers patients $(\mathrm{E}, \mathrm{G})$, while higher mRNA expression of FAM3D was significantly related to poorer OS of thyroid cancer patients(I). However, combinatory mRNA expression of FAM3s showed correlation with DFS in thyroid cancer patients (B). 


\section{A Altered in 78 (16\%) of 497 sequenced cases patients (498 total)}
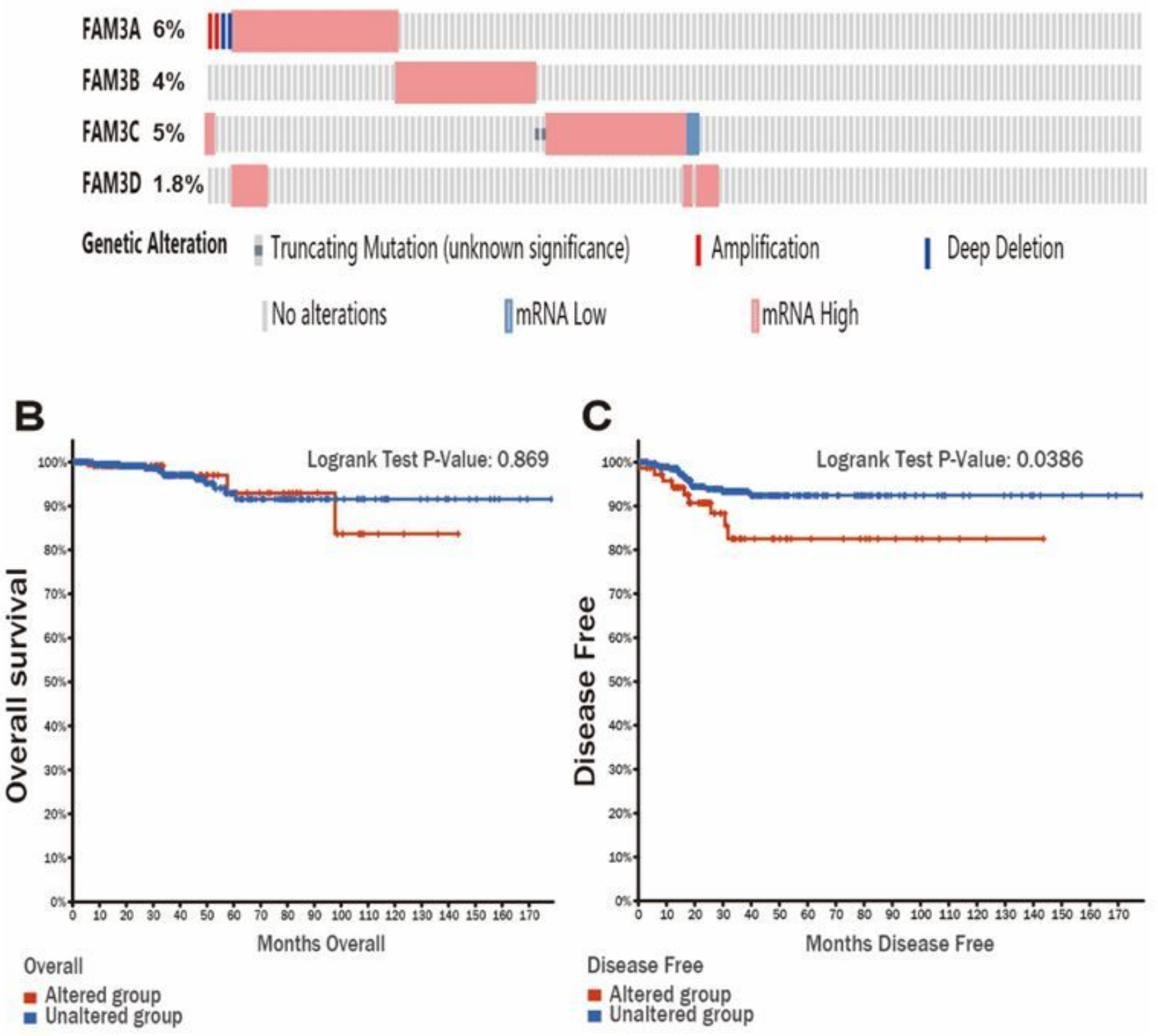

\section{Figure 6}

Genetic mutations in FAM3s and their association with OS and DFS of THCA patients (cBioPortal). Low mutation rate $(16 \%)$ of FAM3s was observed in THCA patients. mutation rates of FAM3A, FAM3B, FAM3C and FAM 3 D were $6 \%, 4 \%, 5 \%$ and $1.5 \%$, respectively (A). 

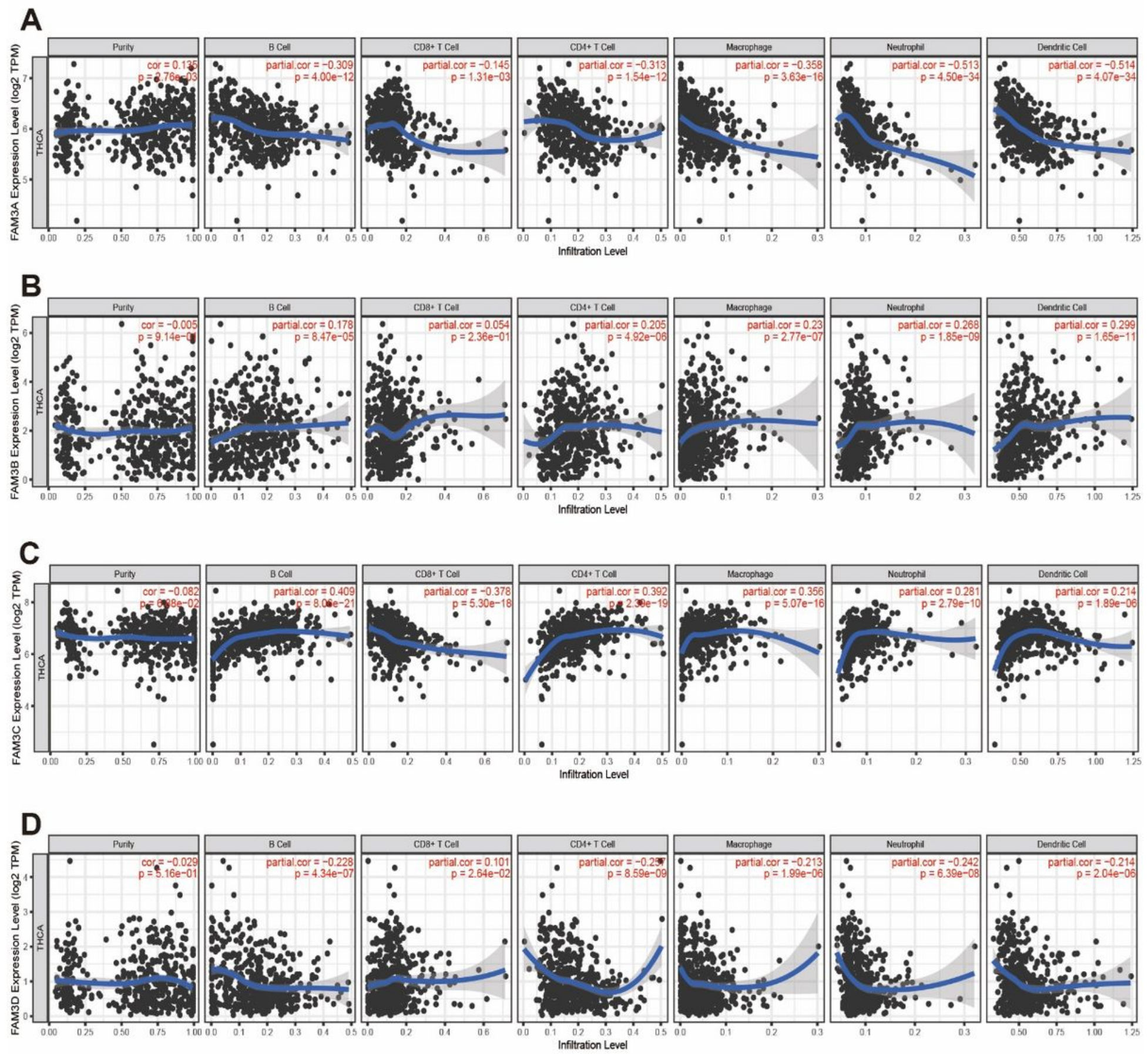

Figure 7

The correlation between different expressed FAM3s and immune cell infiltration (TIMER). The correlation between the abundance of immune cell and the expression of (A) FAM3A, (B) FAM3B, (C) FAM3C, (D) FAM3D in THCA. 